\title{
Effect of glycerol on the penetrating ability of fresh ram spermatozoa with zona-free hamster eggs
}

\author{
T. Slavík \\ The Czechoslovak Academy of Sciences, Institute of Animal Physiology and Genetics, \\ CS-27721 Liběchov, Czechoslovakia
}

\begin{abstract}
Summary. The presence of $10 \%$ glycerol in diluted semen for 30-90 min before preincubation stimulated sperm penetration of zona-free eggs. Prolongation of semen storage in medium with glycerol did not further improve penetration ability. The penetrating activity of spermatozoa depended on the concentration of glycerol and incubation time. These results indicate that the presence of glycerol accelerates induction of the acrosome reaction which could be one of the causes of lower conception rate after insemination with deep-frozen ram semen.
\end{abstract}

\section{Introduction}

It is generally accepted that the lower conception rate achieved when using deep-frozen semen is caused by damage to the spermatozoa in the course of the freezing-thawing procedure, even if a cryoprotectant is used. Glycerol is widely used as a cryoprotective agent and its effect on human spermatozoa has been studied by Tulandi \& McInnes (1984). These authors concluded that glycerol negatively affects sperm motility even before freezing at concentration of $2 \%$ and higher. Jeyendran et al. (1984) compared the cryoprotective effect of glycerol with that of a zwitterion buffer system, TESTCY (mixture of TRIS and TES). Human spermatozoa that were cryopreserved in the presence of glycerol showed greater motility immediately after thawing than did those preserved in the presence of TESTCY. However, after $2 \mathrm{~h}$ in culture, TESTCY-treated spermatozoa possessed higher motility than did glycerol-treated ones. Since the work of Hanada \& Chang (1972) the ability of zona-free hamster eggs to interact with alien spermatozoa in vitro has been used as a test to evaluate sperm fertilizing ability. Jeyendran et al. (1984) assessed the fertilizing potential of frozen human spermatozoa by their ability to fuse with zona-free hamster eggs. After thawing, TESTCY-treated human spermatozoa exhibited better fusion than glycerol-treated spermatozoa. Jeyendran et al. (1985) reported a poor penetration rate of glycerol-treated cryopreserved human spermatozoa in comparison with untreated fresh control semen.

The aim of the present study was to analyse the effect of glycerol upon capacitation and the acrosome reaction in freshly ejaculated ram spermatozoa by assessment of their interaction with zona-free hamster eggs.

\section{Materials and Methods}

Ram spermatozoa and zona-free hamster eggs were allowed to interact as described by Pavlok \& Fléchon (1985). The effect of concentration and of duration of exposure to glycerol, time of preincubation and kinetics of fusion were evaluated.

Mature hamster females were superovulated with injections of 100 i.u. PMSG (Bioveta Ivanovice, Czechoslovakia) per female and 100 i.u. hCG (Praedyn, Spofa Praha, Czechoslovakia) 72-78 h later. At 16-18 h after hCG injection the hamsters were killed and their oocytes isolated from the oviducts. Cumulus oophorus was removed with hyaluronidase (SEVAC Praha, Czechoslovakia) at a concentration of 190 TRU (turbidimetric units)/1 ml culture medium. The zona pellucida was dissolved by $0.02 \%$-chymotrypsin (Koch-Light Laboratories Ltd, Colnbrook, 
U.K.). The details of the method were as described in detail by Pavlok (1981) and Majerčiak et al. (1982). The medium TCM 199 (ÚSOL Praha, Czechoslovakia) used for sperm capacitation, in-vitro fertilization, and dissolving of enzymes, was completed with 0.91 mm-sodium pyruvate (SERVA, Heidelberg, FRG), 3.25 mm-calcium lactate (B.D.H., Poole, U.K.), lyophilized bovine serum proteins, $20 \mathrm{mg} / \mathrm{ml}$ (SEVAC, Praha, Czechoslovakia), 25 i.u. penicillin (K-salt) $/ \mathrm{ml}$, and 25 i.u. streptomycin sulphate $/ \mathrm{ml}$. The pH after equilibration with $5 \% \mathrm{CO}_{2}$ and $95 \%$ air was $7 \cdot 2-7 \cdot 4$.

Spermatozoa were collected with an artificial vagina from 2 rams. After collection each ejaculate was divided into two haives. The first ones were treated as follows.

For the first washing procedure, culture medium enriched with $10 \%$ glycerol (SERVA, Heidelberg, FRG; 5 and 10\% respectively in Exp. 1) was used. After dilution to 1:8-1:10, semen samples were stored for about 10 min (30,60, 90 or $120 \mathrm{~min}$ in Exp. 2) at room temperature and then the samples were centrifuged for $10 \mathrm{~min}$ at $600-800 \mathrm{~g}$. The supernatant was discarded and the washing procedure was repeated once more using culture medium without glycerol. Spermatozoa were preincubated in small amounts of $40-50 \mu \mathrm{l}$ in special microtubes at $37.5^{\circ} \mathrm{C}$ in an humidified atmosphere of $5 \% \mathrm{CO}_{2}$ and $95 \%$ air in Petri dishes. The sperm concentration at that time was $0.4-0.8 \times 10^{9} / \mathrm{ml}$. Preincubation lasted $46 \mathrm{~h}(0,2,4,6,8$ and $10 \mathrm{~h}$ in Exp. 3). Co-incubation of spermatozoa and zona-free eggs was for $16 \mathrm{~h}(1 \cdot 5,3,4 \cdot 5$ or $16 \mathrm{~h}$ in Exp. 4).

The second halves of the ejaculates were treated in the same manner but using culture medium without glycerol for both washings.

At the end of culture, the eggs were mounted under a coverslip supported by two strips of vaseline, fixed with $25 \%$ aceto-alcohol overnight, stained with $2 \%$ aceto-orcein and observed under a Zeiss phase-contrast microscope (Amplival). The criterion for penetration was the identification of swollen sperm heads or male pronuclei with sperm tails in the cytoplasm as described previously by Pavlok \& McLaren (1972). The percentage of penetrated eggs in total number of eggs and the average sperm count in each penetrated egg (polyspermy) were evaluated. Each experiment was repeated at least 3 times.

\section{Results}

In all experiments it was found that glycerol stimulates penetration activity of fresh ram spermatozoa with zona-free hamster eggs.

\section{Experiment 1: effect of glycerol concentration}

The untreated spermatozoa showed lower penetration rates and polyspermy than did glyceroltreated spermatozoa. The penetration activity was greater when medium enriched with $10 \%$ glycerol was used than when the medium contained only $5 \%$ glycerol (Table 1 ).

Table 1. Effect of glycerol concentrations on penetration of zona-free hamster eggs by fresh ram spermatozoa preincubated for $5-6 \mathrm{~h}$

\begin{tabular}{lcc}
\hline $\begin{array}{c}\text { Glycerol } \\
\text { conc. }(\%)\end{array}$ & $\begin{array}{c}\text { Total no. of eggs } \\
(\%) \text { penetrated }\end{array}$ & $\begin{array}{c}\text { Sperm no. per } \\
\text { penetrated egg }\end{array}$ \\
\hline 0 (control) & $116(52 \cdot 6)$ & 1.57 \\
5 & $119(58.0)$ & 1.62 \\
10 & $123(63.4)$ & 2.02 \\
\hline
\end{tabular}

\section{Experiment 2: effect of duration of exposure of spermatozoa to glycerol}

The penetration activity depended on the period of incubation. Glycerol-treated spermatozoa showed the highest penetration activity after incubation for $30 \mathrm{~min}$. Longer periods of storage induced a gradual decrease of penetration activity and after incubation with glycerolated medium for 120 min the penetration activity was lower than in untreated (control) samples (Table 2). 
Table 2. Effect of exposure time to glycerol on penetrating ability of zona-free hamster eggs with fresh ram spermatozoa preincubated for $4-5 \mathrm{~h}$

\begin{tabular}{ccc}
\hline $\begin{array}{c}\text { Exposure time } \\
(\mathrm{min})\end{array}$ & $\begin{array}{c}\text { Total no. of eggs } \\
(\%) \text { penetrated }\end{array}$ & $\begin{array}{c}\text { Sperm no. per } \\
\text { penetrated egg }\end{array}$ \\
\hline 0 (control) & $128(28 \cdot 9)$ & $1 \cdot 32$ \\
30 & $83(79 \cdot 5)$ & $1 \cdot 82$ \\
60 & $83(74 \cdot 7)$ & $1 \cdot 73$ \\
90 & $80(53 \cdot 6)$ & $1 \cdot 53$ \\
120 & $81(22 \cdot 2)$ & $1 \cdot 16$ \\
\hline
\end{tabular}

Table 3. Effect of glycerol on the penetrating ability of fresh ram spermatozoa preincubated for different times

\begin{tabular}{lccc}
\hline $\begin{array}{c}\text { Glycerol } \\
\text { conc. }(\%)\end{array}$ & $\begin{array}{c}\text { Time of } \\
\text { preincubation } \\
(\mathrm{h})\end{array}$ & $\begin{array}{c}\text { Total no. } \\
\text { of eggs } \\
(\%) \text { penetrated }\end{array}$ & $\begin{array}{c}\text { Sperm no. per } \\
\text { penetrated egg }\end{array}$ \\
\hline 0 (control) & 0 & $142(2 \cdot 1)$ & 1.00 \\
& 2 & $138(14 \cdot 5)$ & 1.30 \\
& 4 & $137(36.5)$ & 1.50 \\
& 6 & $134(55 \cdot 2)$ & 1.77 \\
& 8 & $137(37 \cdot 2)$ & 1.69 \\
10 & 10 & $131(26.0)$ & 1.59 \\
& 0 & $145(22.8)$ & $1 \cdot 15$ \\
& 2 & $137(48.9)$ & 1.58 \\
& 4 & $135(68 \cdot 1)$ & 2.22 \\
& 6 & $142(45 \cdot 1)$ & 1.97 \\
& 8 & $133(19.5)$ & 1.50 \\
& 10 & $138(5.8)$ & 1.25 \\
\hline
\end{tabular}

Table 4. Effect of glycerol on the kinetics of zona-free hamster egg penetration with fresh ram spermatozoa preincubated for 4-5h

\begin{tabular}{lccc}
\hline $\begin{array}{c}\text { Glycerol } \\
\text { conc. (\%) }\end{array}$ & $\begin{array}{c}\text { Sperm-egg } \\
\text { incubation } \\
\text { (h) }\end{array}$ & $\begin{array}{c}\text { Total no. } \\
\text { of eggs } \\
\text { (\%) penetrated }\end{array}$ & $\begin{array}{c}\text { Sperm no. per } \\
\text { penetrated egg }\end{array}$ \\
\hline 0 (control) & 1.5 & $110(26 \cdot 4)$ & $1 \cdot 14$ \\
& 3.0 & $110(36 \cdot 4)$ & 1.18 \\
& 4.5 & $102(44 \cdot 1)$ & 1.31 \\
10 & 16.0 & $107(72 \cdot 9)$ & 1.85 \\
& 1.5 & $108(64 \cdot 8)$ & 1.46 \\
& 3.0 & $111(82 \cdot 9)$ & 1.92 \\
& 4.5 & $110(91 \cdot 8)$ & 2.31 \\
& 16.0 & $103(94 \cdot 2)$ & 2.54 \\
\hline
\end{tabular}


Experiment 3: effect of glycerol on the ability of ram spermatozoa to penetrate zona-free hamster eggs

Glycerol reduced the time necessary for preincubation and glycerol-treated spermatozoa gained the ability to penetrate zona-free eggs earlier. Spermatozoa that were untreated and not preincubated penetrated at a very low level. Maximal activity was achieved after $6 \mathrm{~h}$ preincubation and the penetrating ability was still present after preincubation for $10 \mathrm{~h}$. When the spermatozoa were treated with glycerol, penetration occurred without preincubation, and maximal penetration was after $4 \mathrm{~h}$ preincubation. However, the decrease of penetrating ability occurred earlier and after preincubation for $10 \mathrm{~h}$ glycerol-treated spermatozoa showed very low penetration (Table 3 ).

\section{Experiment 4: effect of glycerol on kinetics of penetration}

Untreated spermatozoa penetrated the zona-free eggs gradually during the culture $(16 \mathrm{~h})$, while glycerol-treated spermatozoa penetrated mostly during the first $4.5 \mathrm{~h}$ of culture. This was deduced from differences between values after $4.5 \mathrm{~h}$ and $16 \mathrm{~h}$. For untreated spermatozoa the difference was $28.8 \%(72.9-44.1 \%)$ penetrated eggs and $0.54(1.85-1.31)$ spermatozoa per egg were calculated. For glycerol-treated spermatozoa the equivalent differences were $2 \cdot 4 \%(94 \cdot 2-91 \cdot 8 \%)$ penetrated eggs and $0 \cdot 23(2 \cdot 54-2 \cdot 31)$ spermatozoa per egg (Table 4$)$.

\section{Discussion}

After glycerol treatment the absolute number of ram spermatozoa that were able to penetrate zona-free hamster eggs increased in a very short interval. It can be therefore supposed that glycerol accelerates and synchronizes the induction of the acrosome reaction.

One of the factors which could affect the course of capacitation and the acrosome reaction is the binding of fatty acids to glycerol, although there is no direct evidence for this possibility. Free fatty acids function as a decapacitator and after their removal (e.g. by binding to albumin) the sperm membranes are destabilized and the acrosome reaction induced (Lui \& Meizel, 1977; Aonuma et al. 1982; Juetten \& Bavister, 1983). For free fatty acids binding to glycerol the equilibrium of reaction 'membrane phospholipids $\rightarrow$ lysophospholipids + free fatty acids' is destroyed and lysophospholipids, several of which function as guinea-pig acrosome reaction-stimulating factors (Fleming \& Yanagimachi, 1981), could better be applied.

In view of the widespread use of glycerol in diluents for deep freezing of semen, it is of interest that ram spermatozoa can utilize it under aerobic conditions. According to Hutton et al. (1980) and Jones \& Stevenson (1983), glycerol could be linked to glycolytic pathways at the triose phosphate stage. Triose phosphates are converted into lactic acid which can be further oxidized. These steps require oxygen and therefore glycerol stimulates respiration in semen of rams (Mann \& White, 1957) and bulls (Halangk et al., 1985). Regarding the induction of the acrosome reaction, the metabolic pathway is less certain, because an increase of penetration ability is apparent even after a short incubation.

The acrosome reaction could also be stimulated by osmotic pressure changes of medium, because the osmotic pressure of the culture medium increased several times after glycerol addition. A stimulatory effect of hyperosmolarity on the acrosome reaction of human spermatozoa has been shown by Aitken et al. (1983) and Oliphant \& Brackett (1973) concluded that glycerol affects negatively sperm motility and fertilization of intact mouse ova.

In the light of the present results it seems that a lower conception rate after using frozen-thawed semen could be caused by acceleration of the capacitation events. Crozet \& Dumont (1984) suggest that the acrosome reaction occurs at the surface of the zona pellucida in ewes. Hunter et al. (1980) detected spermatozoa in sheep oviducts 6-8 h after insemination. Hunter \& Nichol (1983) found fertilized eggs $22-24 \mathrm{~h}$ after mating at the onset of oestrus. At least for some of the cryopreserved 
spermatozoa therefore the acrosome reaction could be induced earlier, perhaps in a reservoir of the female genital tract even before ovulation and so spermatozoa could be released as fully acrosomereacted or the acrosome reaction is achieved during the passage through the female genital tract. It may be for this reason that Salamon \& Lightfoot (1967), using frozen-thawed ram semen, obtained a higher pecentage of fertilized eggs after intrauterine insemination than after cervical insemination. Similarly, Armstrong \& Evans (1984) achieved higher conception rates after intrauterine insemination with deep-frozen ram semen than after cervical insemination.

Further experiments concerning assessment of the fertilizing ability of glycerol-treated ram spermatozoa in vivo in relation to the time of ovulation are in progress.

I thank Dr Antonin Pavlok and Dr Josef Fulka for their valuable comments and suggestions; and Dr František Zavřel for help in preparing the manuscript and editing the text.

\section{References}

Aitken, R.J., Wang, Y.F., Liu, J., Best, F. \& Richardson, D.W. (1983) The influence of medium composition, osmolarity and albumin on acrosome reaction and fertilizing capacity of human spermatozoa: development of an improved zona-free hamster egg penetration test. Int. J. Androl. 6, 180-193.

Aonuma, S., Okabe, M., Kishi, Y., Kawaguchi, M. \& Yamada, H. (1982) Capacitation inducing activity of serum albumin in fertilization of mouse ova in vitro. J. Pharm. Dynam. 5, $980-987$.

Armstrong, D.T. \& Evans, G. (1984) Intrauterine insemination enhances fertility of frozen semen in superovulated ewes. J. Reprod. Fert. 71, 89-94.

Crozet, N. \& Dumont, M. (1984) The site of the acrosome reaction during in vivo penetration of the sheep oocyte. Gamete Res. 10, 97-105.

Fleming, A.D. \& Yanagimachi, R. (1981) Effects of various lipids on the acrosome reaction and fertilizing capacity of guinea pig spermatozoa with special reference to the possible involvement of lysophospholipids in the acrosome reaction. Gamete Res. 4, 253-273.

Halangk, W., Bohnensack, R., Frank, K. \& Kunz, W. (1985) Effect of various substrates on mitochondrial and cellular energy state of intact spermatozoa. Biomed. Biochim. Acta 44, 411-420.

Hanada, A. \& Chang, M.C. (1972) Penetration of the zona-free eggs by sperm of different species. Biol. Reprod. 6, 300-309.

Hunter, R.H.F. \& Nichol, R. (1983) Transport of spermatozoa in the sheep oviduct: preovulatory sequestering of cells in the caudal isthmus. J. exp. Zool. 228, 121-128.

Hunter, R.H.F., Nichol, R. \& Crabtree, S.M. (1980) Transport of spermatozoa in the ewe: timing of establishment of a functional population in the oviduct. Reprod. Nutr. Develop. 20, 1869-1875.

Hutton, P., Dawson, A.G. \& Jones, A.R. (1980) Inhibition of glycolysis in boar sperm by $\alpha$-chlorohydrin. Contraception 22, 505-512.

Jeyendran, R.S., Van der Ven, H.H., Kennedy, W., PerezPelaez, M. \& Zaneveld, L.J.D. (1984) Comparison of glycerol and a zwitter ion buffer system as a cryoprotective medium for human spermatozoa. J. Androl. 5, $1-7$.
Jeyendran, R.S., Van der Ven, H.H., Perez-Pelaez, M. \& Zaneveld, L.J.D. (1985) Effect of glycerol and cryopreservation on oocyte penetration by human spermatozoa. Andrologia 17, 241-248.

Jones, A.R. \& Stevenson, D. (1983) Formation of active antifertility metabolite of (S)- $\alpha$-chlorohydrin in boar sperm. Experientia 39, 784-785.

Juetten, J. \& Bavister, B.D. (1983) The effect of amino acids, cumulus cells and bovine serum albumin on in vitro fertilization and first cleavage of hamster eggs. J. exp. Zool. 227, 487-490.

Lui, C.W. \& Meizel, S. (1977) Biochemical studies of the in vitro acrosome reaction inducing ability of bovine serum albumin. Differentiation 9, 59-66.

Majerčiak, P., Pavlok, A. \& Babuśík, P. (1982) Evaluation of the fertilizing ability of fresh boar ejaculate by the test of sperm penetration into Syrian hamster eggs without zona pellucida. Żivočišná výroba 27 , 627-634 (In Slovak).

Mann, T. \& White, I.G. (1957) Glycerol metabolism by spermatozoa. Biochem. J. 65, 634-637.

Oliphant, G. \& Brackett, B.G. (1973) Capacitation of mouse spermatozoa in media with elevated ionic strength and reversible decapacitation with epididymal extracts. Fert. Steril. 24, 948-955.

Pavlok, A. (1981) Penetration of hamster and pig zonafree eggs by boar ejaculated spermatozoa preincubated in vitro. Int. J. Fert. 26, 101-106.

Pavlok, A. \& Fléchon, J.E. (1985) Some factors influencing the interaction of ram spermatozoa with zona-free hamster eggs. J. Reprod. Fert. 74, 597-604.

Pavlok, A. \& McLaren, A. (1972) The role of cumulus cells and the zona pellucida in fertilization of mouse eggs in vitro. J. Reprod. Fert. 29, 91-97.

Salamon, S. \& Lightfoot, R.J. (1967) Fertilization and embryonic loss in sheep after insemination with deep frozen semen. Nature, Lond. 216, 194-195.

Tulandi, T. \& McInnes, R.A. (1984) Vaginal lubricants: Effects of glycerol and egg white on sperm motility and progression in vitro. Fert. Steril. 41, 151-153. 